\title{
DAMAGE DETECTION IN CFRP PLATES BY MEANS OF NUMERICAL MODELING OF LAMB WAVES PROPAGATION
}

\author{
Grzegorz Knor ${ }^{1}$ \\ ${ }^{1}$ Institute of Fundamental Technological Research Polish Academy of Sciences, Warsaw, Poland
}

\begin{abstract}
The paper presents an application of modeling acoustic waves propagation in a carbon fiber reinforced plastic (CFRP) plates for damage detection. This task is a part of non-destructive testing (NDT) methods which are very important in many industry branches. Propagation of Lamb waves is modeled using three-dimensional finite element method by means of commercial software. In the paper three different cases of plate structures with and without flaws are considered to present review of selected methods for the detection of defects in time and frequency domain. These are comparisons of: A-scans, B-scans, dispersion curves, spectrograms, scalograms and energy plots. Developed numerical model first has been validated by means of analytical solution for isotropic plate.
\end{abstract}

Keywords: Lamb waves, non-destructive testing, finite element method, damage detection

\section{INTRODUCTION}

Many of construction elements are made of composite materials (e.g. plane's wings, components of cars coachwork, boats, wind turbines blades [1]) and because of importance of these elements they must be tested by means of NDT techniques during manufacturing and exploitation. The main reason to do that is to ensure the reliability of the whole constructions made of CFRP materials. One of the ways to detect flaws in plate structures are techniques based on so called Lamb wave propagation [2]. The mathematical background for this phenomenon was developed by Horace Lamb in 1917 [3] and since 1980s Lamb waves have been successfully used in Structural Health Monitoring and Nondestructive evaluation [4]. Theoretical models and experimental methods are still being developed mainly because of their advantages. In his classical paper Lamb developed only equations for waves propagation in isotropic objects of simplified geometries [3]. CFRP belongs to a class of anisotropic materials and very often structures made of it have complicated geometry (curved shape, presence of stiffeners or joints). Due to the complexity there is no analytical solution in such cases. Moreover these phenomena become more complex with the presence of a flaw, because defects in a structure interfere with propagating waves. To deal with these problems many algorithms have therefore been developed for the effective damage detection with the aid of advanced signal processing and identification approaches [4], viz. Fourier transform, wavelet analysis, dispersion curves, $\mathrm{A}$ and B-scans comparisons, etc. Furthermore due to lack of the analytical solution for a better understanding of Lamb waves phenomena in layered materials there is need to develop accurate numerical models. A number of different numerical computational techniques have been developed and can be used for this type of analysis bearing in mind their limitations. For example according to the literature following algorithms can be used: finite difference method
(FDM) [5], finite element method (FEM) [6], finite strip method (FSM) [7], boundary element method (BEM) [8], global matrix approach [9], spectral element method (SEM) [10], mass-spring lattice model [11]. The most commonly used technique seems to be a higher order FEM, which utilizes Lagrangian shape functions on a Gauss-LobattoLegendre grid (it is called spectral-element method in the literature). But SEM is also limited. For example algorithm developed by Ostachowicz et.al. [10] gives very good approximation only for fundamental modes of Lamb waves. For example for a $1 \mathrm{~mm}$ thick plate made of aluminum alloy both the $s_{0}$ and $a_{0}$ modes can be well represented in the frequency range up to about $500 \mathrm{kHz}$, which constitutes the upper limit of practical application of the Reissner-Mindlin theory [10]. In this paper standard FEM is used because of its versatility and wide availability of both commercial and free software. The advantages of FEM include the ability to study Lamb wave propagation almost in any kind of the structure including geometry inhomogeneities, defects, etc. But main limitation of this method is long calculation time and also FEM puts a heavy workload on the CPU and memory. Problems increases with frequency increment (frequencies are in hundreds of kilohertz range [12]), because sampling step in space should be not greater than $20 \lambda$ ( $\lambda$ is a wave lengths) [13] and sampling step in time should satisfy Nyquist-Shannon sampling theorem [14].

\section{MATHEMATICAL BACKGROUND}

The wave propagation in stress-free isotropic and anisotropic bulk media can be adequately described using theory based on linear stress-strain relationships. The stress equation of motion [15]:

$\sigma_{i j, j}+\rho f_{i}=\rho \ddot{u}_{i}$ 
Hooke's law:

$$
\sigma_{i j}=C_{i j k l} \varepsilon_{k l}
$$

And linear strain-displacement relationship:

$\varepsilon_{i j}=\frac{1}{2}\left(u_{i, j}+u_{j, i}\right)$

where $\sigma$ is the Cauchy stress tensor, $\varepsilon$ is the Cauchy strain tensor, $\rho$ is the material density, $u$ is the displacement, $f$ is a vector of the external force, $C$ is fourth-order stiffness tensor and two dots over a letter denote the second order derivative with respect to time. Einstein summation convention and a comma derivative notation are used. Combining the Eqs. 1-3 one can write the following system of equations:

$$
C_{i j k l} u_{k, j l}=\rho \ddot{u}_{i}
$$

Differential equations of motion given by Eq. 4 with associated boundary conditions can be solved analytically only for limited number of cases. On the whole the geometry, boundary conditions and potential defects complicate the situation, that the only possible solution is to use the numerical model. One of the example where a semianalytical solution exists is the propagation of Lamb waves in thin isotropic plate (for example made of aluminum). In this case the elastic oscillations are described by the Rayleigh-Lamb equations:

$$
\frac{\tan (p d)}{\tan (q d)}=-\left[\frac{4 k^{2} p q}{\left(k^{2}-q^{2}\right)^{2}}\right]^{ \pm 1}
$$

where:

$$
p^{2}=\frac{\omega^{2}}{c_{L}^{2}}-k^{2}
$$

And

$$
q^{2}=\frac{\omega^{2}}{c_{T}^{2}}-k^{2}
$$

$k$ is the wave number, $\omega$ is the angular frequency, $c_{L}$ is the longitudinal phase velocity, $c_{T}$ is the shear phase velocity and $d$ is a half thickness of the plate. Eq. 5 can be solved by means of numerical software according to the algorithm proposed in [15]. The solution of Eq. 5 is the dispersion relation between the wave number and angular frequency. Fig. 1 presents dispersion curves (symmetrical modes $s_{0}, s_{l}$, $s_{2}$ and antisymmetric modes: $\left.a_{0}, a_{1}\right)$ in k- $\omega$ representation for $3 \mathrm{~mm}$ thick aluminum plate. To plot result in more common than $k$ - $\omega$ form one need to perform transformation to $f d-v_{p}$ representation by calculating the phase velocity $v_{p}$, which is defined as the following quotient:

$v_{p}=\frac{f}{k}$

Where $f=2 \pi \omega-$ frequency and $k$ is a wave number.

\section{NUMERICAL MODEL}

\subsection{Model Details}

The plate was modelled by means of Structural Mechanic Module in COMSOL Multiphysics software as a linear elastic material. To achieve good numerical accuracy at least

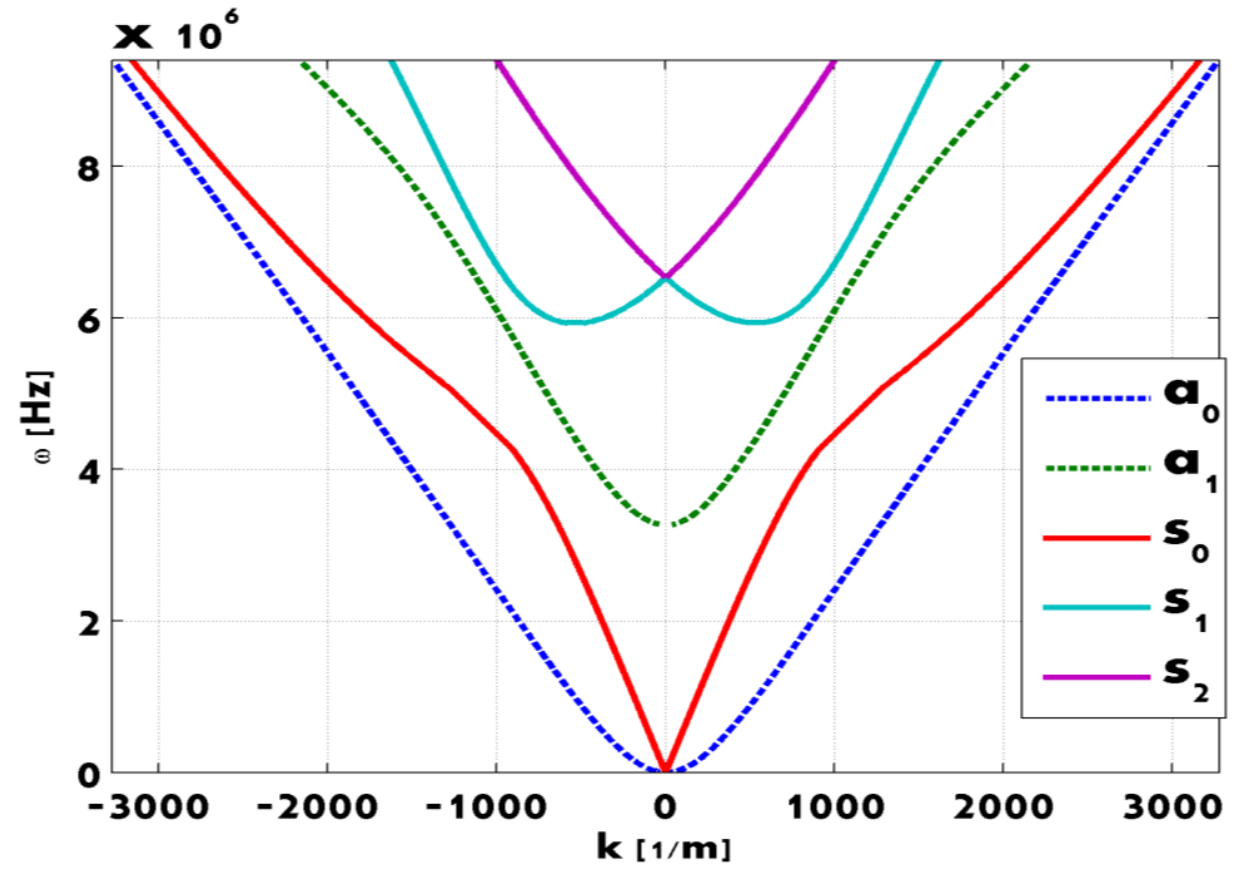

Fig- 1: Calculated dispersion curves (symmetrical and antisymmetric modes) - k- $\omega$ representation. 
5 second-order mesh elements per wavelength are required [16] (some authors propose more rigorous condition [13]). So the maximum allowed mesh element size becomes:

$$
h_{0}=\frac{c}{N f_{0}}
$$

where $c$ is the local speed of the wave, and $N=5$ is the number of mesh elements per wavelength. Also time step is connected with the accuracy issue and element size. The relationship between mesh size and time step length is known as the Courant-Friedrichs-Lewy condition [17] and it can be expressed as:

$$
C F L=c \cdot \Delta t / h
$$

where $\Delta t$ is the time step and $h$ is the mesh size. In practice, with second order mesh elements, a CFL number of 0.2 is chosen as optimal. In discussed simulation time step $\Delta t$ is equal 100ns and it makes that CFL condition is satisfied. As excitation sinusoidal signals with a rectangular window or Hanning window are usually used in nondestructive using Gaussian distribution (see Fig. 2). The mathematical evaluation and structural health monitoring. These signals are usually called a tone burst. In this paper a 3-cycle tone burst at $200 \mathrm{kHz}$ center-frequency is used. The $N_{c}$ cycle Hanning windowed tone burst at given frequency can be expressed as:

$$
f(t)=0.5\left[1-\cos \left(\omega_{0} t / N_{c}\right)\right] \sin \left(\omega_{0} t\right)
$$

for time in the range of:

$$
t \in\left\langle 0, N_{c} / f_{0}\right\rangle
$$

Where: $f_{0}-$ center frequency, $N_{c}$ - number of cycles, $\omega_{0}-$ center angular frequency, $t-$ time.

Another remark refers to the excitation of a plate. In this work the properties of transmitter are ignored and interaction between transmitter and plate are modeled as surface load and distributed over the area of transmitter form of Gaussian distribution in this case is as follows:



Fig - 2: Schematic representation of the transmitter and emitted beam

$$
g(x)=\exp \left(-0.5\left(\frac{x-\text { load_position }}{\text { load_pulse_width }}\right)^{2}\right)
$$

By multiplying surface tone burst (given by Eq. 11) by function $g$ in two directions (e.g. $x$ and $y$ ) one can obtain desired pulse. For completeness of description it should be added that all boundary condition were set to free. It means that there are no constraints and no loads (except surface covered by the transmitter) acting on the boundary. And all initial values (displacements and velocities) were set to 0 .

\subsection{Model Validation}

As it was mentioned the implemented model was validated by means of analytical solution for thin isotropic plate. The thickness of the plate was $3 \mathrm{~mm}$ and for the purposes of calculations the material constants for aluminum were adopted. The results of FEM calculation can be presented in many ways. One of it, so called B-scan, is presented in Fig. 3. 


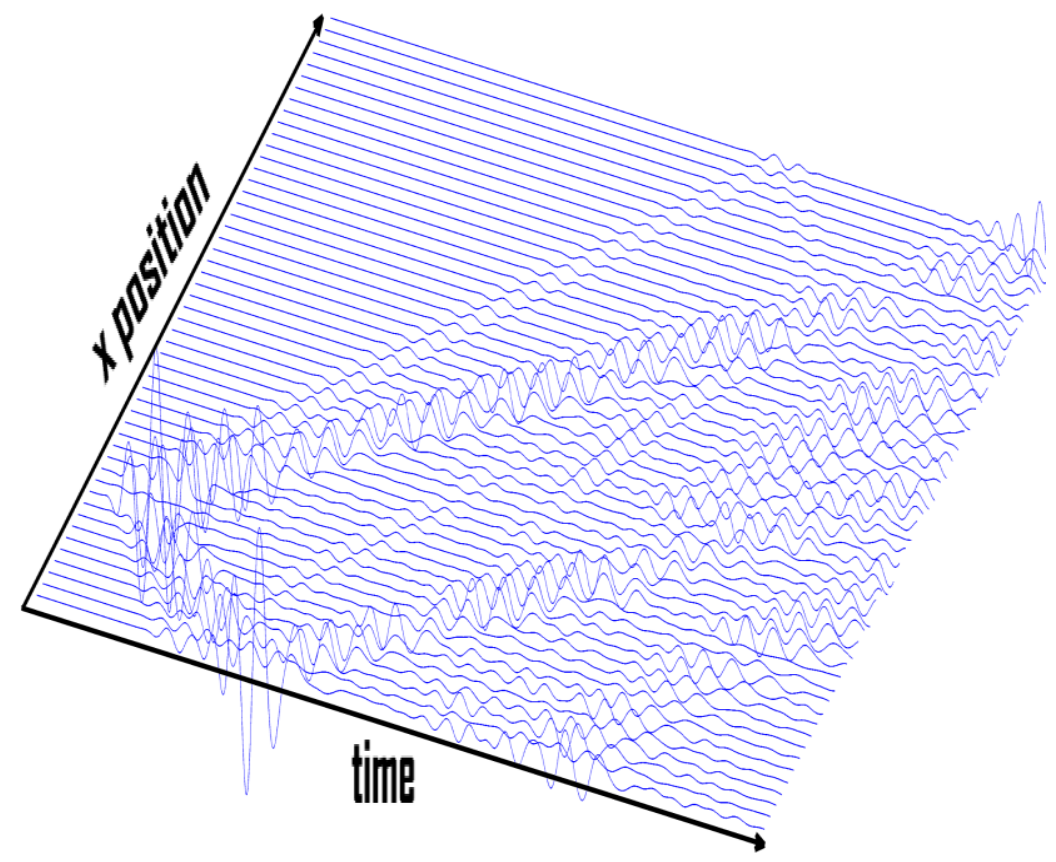

Fig - 3: B-Scan for isotropic aluminum plate

In the B-scan presentation wave amplitudes excited by the same source are recorded at equidistant points lying along a straight line and are displayed with respect to time (x axis) and receivers positions ( $\mathrm{y}$ axis). Based on data stored in matrix like in B-scan presentation dispersion curves can be calculated by means of double Fourier transform: first along "time direction" (transformation from time domain to frequency domain) and next along "space direction" (transformation from spatial domain to wave number domain) [4, 18]. Result is shown in Fig. 4. Comparing the obtained result with the analytical solution shown in Fig. 1 it can be observed that both $a_{0}$ and $s_{0}$ modes are reproduced with good accuracy. The angular frequency-wavenumber representation shows also clear distinction among basic Lamb wave modes. The transformation of result to $f d-v_{p}$ representation is depicted in Fig. 5. Calculated $a_{0}$ and $s_{0}$ modes are plotted on the determined analytically curves. Maximum intensity of the energy is around $600 \mathrm{Hzm}$, which is equal to the product of the excitation frequency and plate thickness. Compliance of the model with the analytical solution allows to use it as a tool for the calculation of wave propagation in plates made of CFRP.

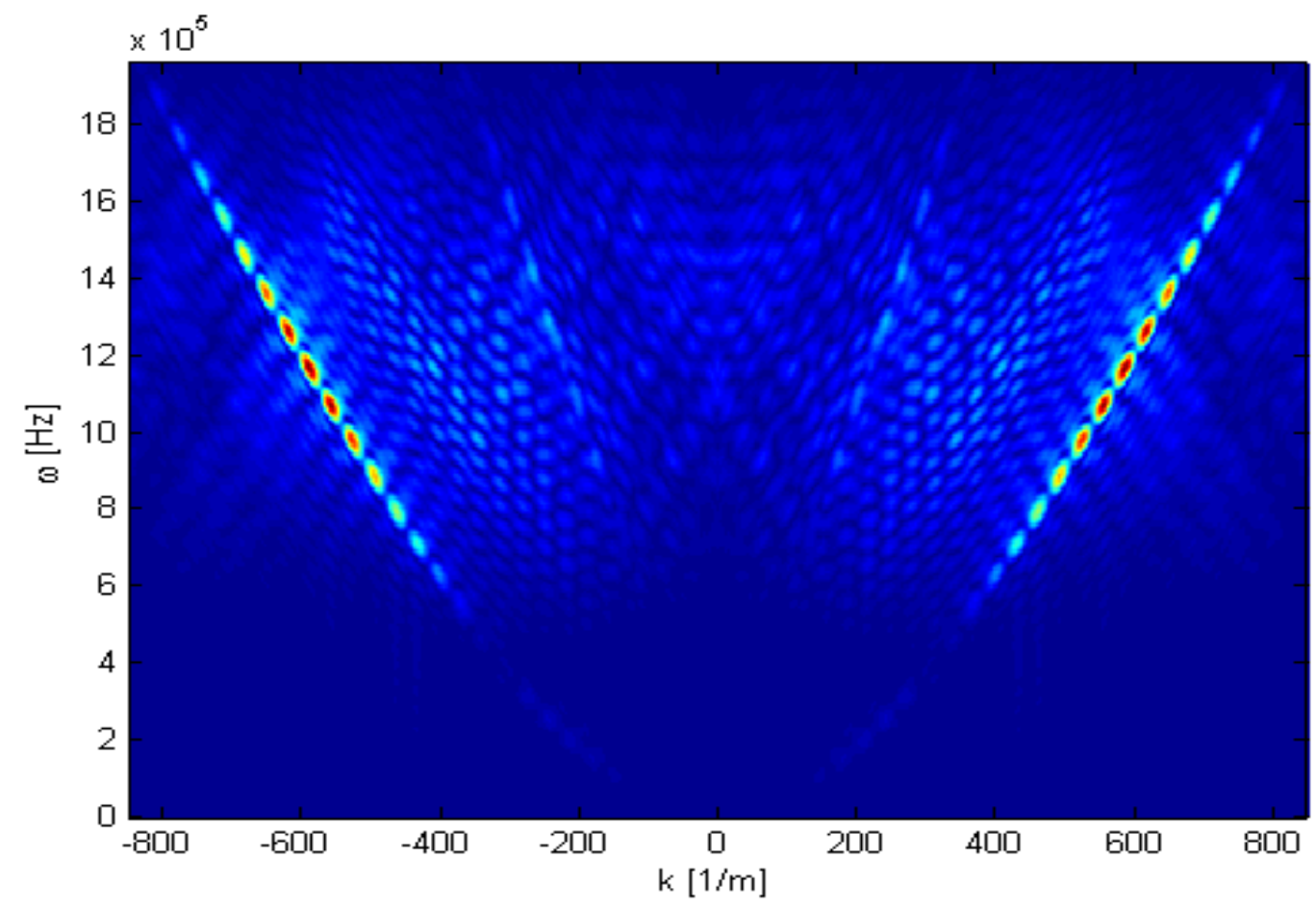

Fig - 4: Dispersion curves for aluminum plate $-k-\omega$ representation 


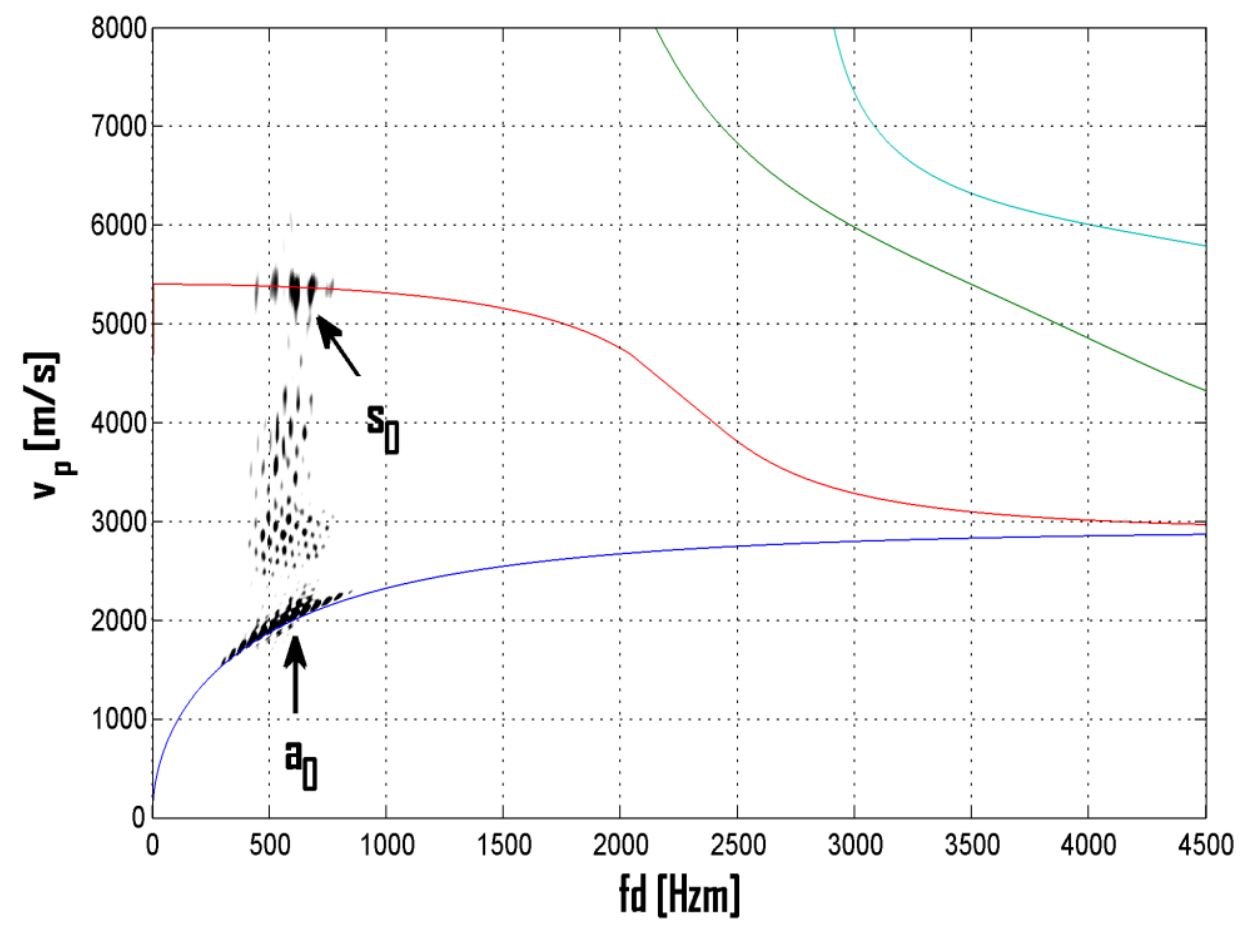

Fig- 5: Dispersion curves for aluminum plate $-f d-v$ representation

\section{RESULT}

\subsection{Numerical Simulation Details}

The results of full-scale models for CFRP T800/913 (unidirectional) plates are presented. The comparison of three cases will be discussed:

- $\quad$ plate without defect - Fig. 6, it is a reference plate,

- $\quad$ plate with small hole located $50 \mathrm{~mm}$ from the middle of the plate in $\mathrm{x}$ direction - Fig. 7,

- $\quad$ plate with defect (cavity) located $50 \mathrm{~mm}$ from the middle of the plate in $\mathrm{x}$ direction on the bottom surface - Fig. 8 .

The thickness of each plate is $1 \mathrm{~mm}$, and the length of edge is $400 \mathrm{~mm}$ (plates are square). Due to the symmetry only one quarter of each plate was analyzed by means of FEM simulation. The excitation pulse was three cycle tone burst modulated by Hanning window with central frequency $200000 \mathrm{~Hz}$. The load was put on the top surface in the middle of the plate. Because of symmetric boundary conditions in case 2 and 3 there are two symmetrically placed flaws. The elastic properties used for FEM simulations were as follows [19]:

$D=\left[\begin{array}{cccccc}154 & 3.7 & 3.7 & 0 & 0 & 0 \\ 3.7 & 9.5 & 5.2 & 0 & 0 & 0 \\ 3.7 & 5.2 & 9.5 & 0 & 0 & 0 \\ 0 & 0 & 0 & 2.5 & 0 & 0 \\ 0 & 0 & 0 & 0 & 4.2 & 0 \\ 0 & 0 & 0 & 0 & 0 & 4.2\end{array}\right] \mathrm{GPa}$
Time of simulation was set to $100 \mu$ s and time step to $100 \mathrm{~ns}$. In each case different meshes were used:

- $\quad$ case 1 (Fig. 6): Number of degrees of freedom solved for: 1447209. Complete mesh consisted of 40000 hexahedral elements. Minimum element quality is 1 ,

- $\quad$ case 2 (Fig. 7): Number of degrees of freedom solved for: 1693791. Complete mesh consisted of 93698 prism elements. Average element quality is 0.99 and minimum element quality is 0.6 ,

- $\quad$ case 3 (Fig. 8): Number of degrees of freedom solved for: 2173890. Complete mesh consisted of 399056 tetrahedral elements. Average element quality is 0.65 and minimum element quality 0.09 .

Where mesh quality is a dimensionless quantity between 0 and 1 , where 1 represents a perfectly regular element, and 0 represents a degenerated element.

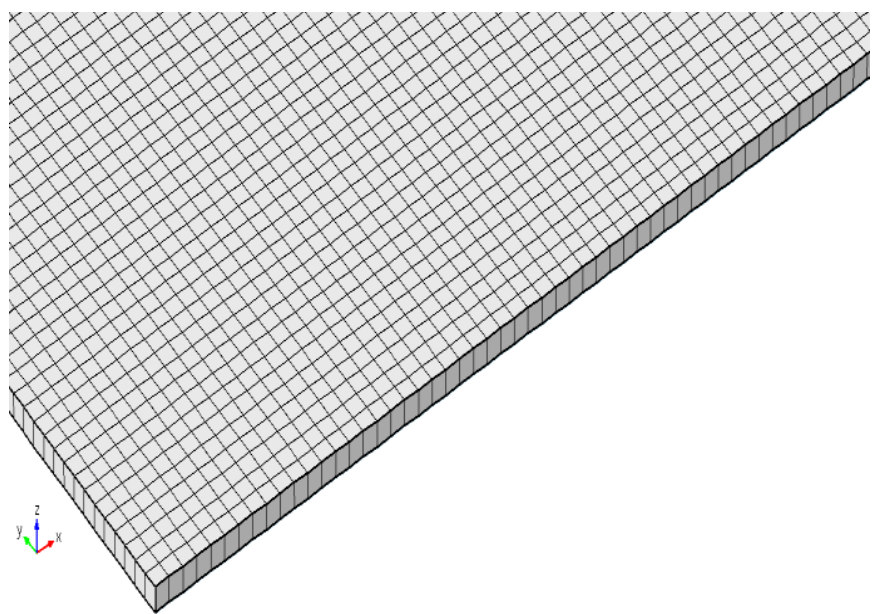

Fig - 6: CFRP plate without damage, hexahedral mesh 


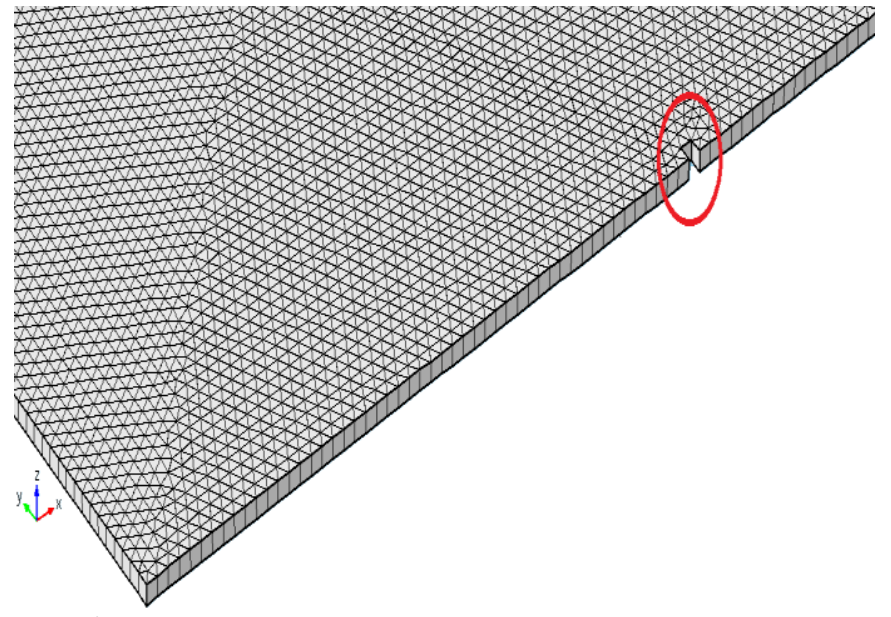

Fig - 7: CFRP plate with damage (hole), prism mesh

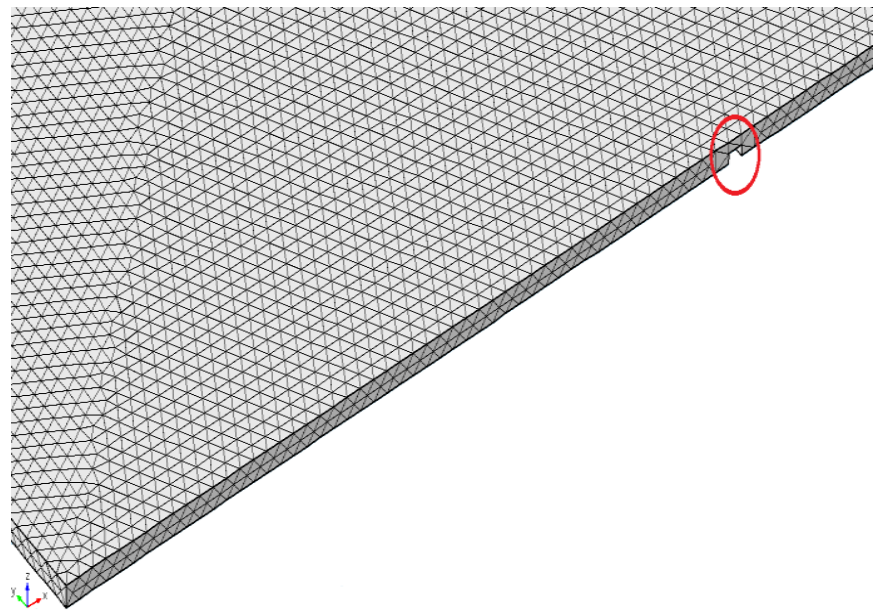

Fig - 8: CFRP plate with damage (cavity), tetrahedral mesh measures the regularity of the mesh elements' shapes, is the best for case 1. But it is important to bear in mind that not every geometry (topology) can be meshed with this type of elements and as a result one has to use tetrahedral elements, which are more flexible.

\subsection{A-Scans}

A convenient way to show differences between recorded (modeled) signals is amplitude representation (so called A-scan). In Fig. 9 such comparison is shown for signals recorded in point $\mathrm{x}=0 \mathrm{~mm}, \mathrm{y}=100 \mathrm{~m}$ (measured from the middle of the plate) on the top surface. Curves plotted in Fig. 9 allow to distinguish between reference plate and plate with defect (in this case whole). But during numerical experiments it was noticed that the choice of location of the sensor has a big impact on the possibility of a detection of defect. So to increase the probability of flaw detection many measurements should be made at different receiver positions.

\subsection{B-Scans}

By making several measurements along a straight line one can obtain mentioned earlier B-scan. In such presentation it is easier to observe the reflection from obstacles than in case of A-scan. The comparison of the three discussed cases of propagation of Lamb waves in anisotropic CFRP plates with and without defects is depicted in Fig. 10. Reflections from obstacles are visible in second and third column (plate with hole and cavity, respectively) for time greater than $40 \mu \mathrm{s}$. Obviously reflections are definitely better visible in case 2, because of the size of hole. Based on such graphical presentation a surveyor, besides the information that there is any fault, can also determine the position of the flaw.

It is clearly seen that hexahedral elements are the best one in this case. First of all number of degrees of freedom is lower for that type of elements. Also mesh quality, which

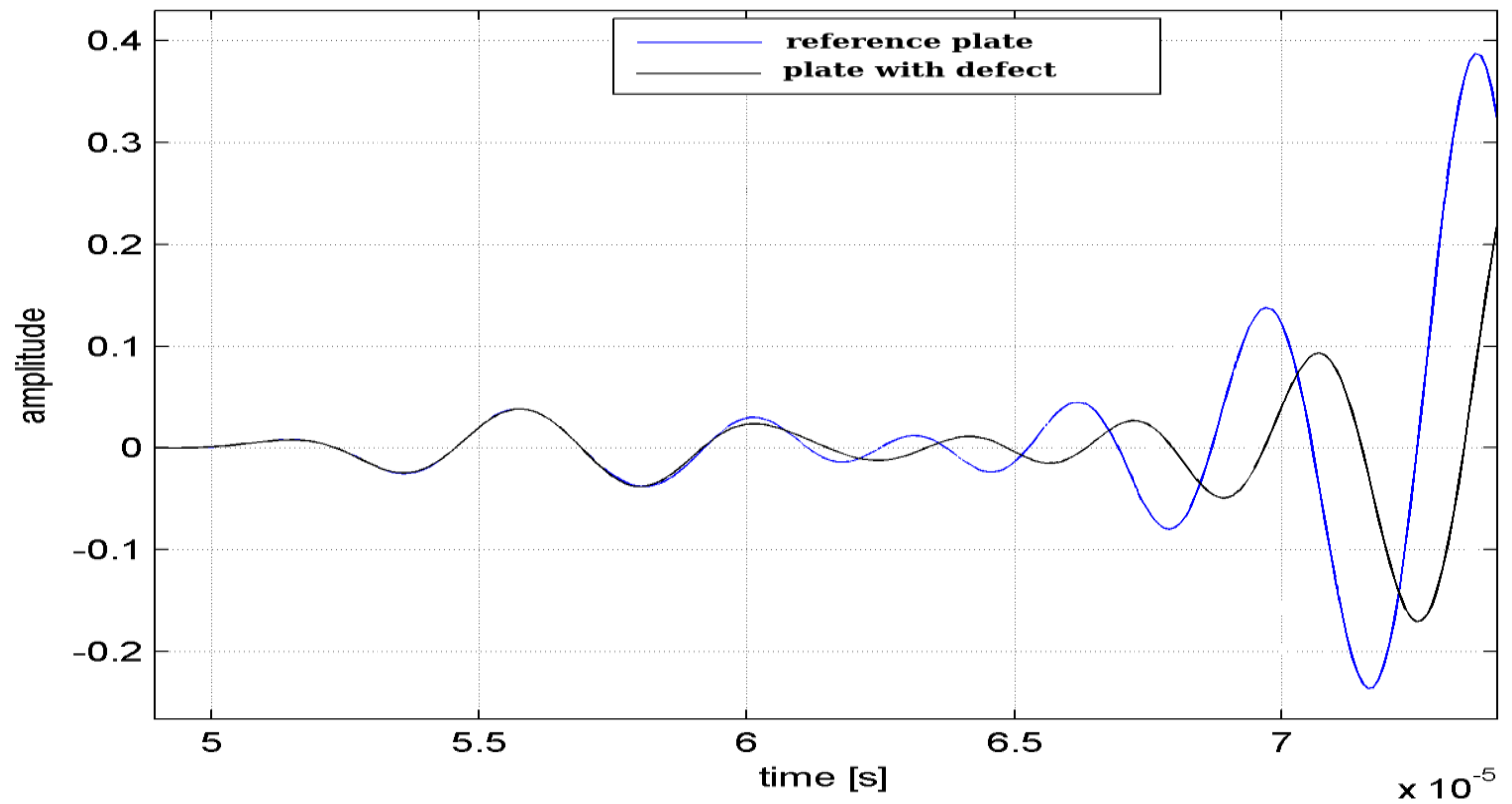

Fig - 9: Comparison of amplitudes for reference plate and plate with hole. Receiver position $\mathrm{x}=0 \mathrm{~mm}, \mathrm{y}=100 \mathrm{~mm}$ 


\subsection{Dispersion Curves}

Another advantage of multiple measurements is a possibility to calculate dispersion curves, which also require a series of measurement from equispaced sensors along the straight line. Fig. 11 presents a comparison of selected model results for CFRP plates. In the left column results for undamaged plate were depicted and in the right column results for plate with hole (case 2) are shown. Angles denote the slope of the line along which the virtual sensors were arranged relative to the coordinate system. It can be observed that pictures for damaged plate (for each angle) are ragged. Unfortunately by means of this type of analysis there is no possibility to identify position of flaw - the spatial information is lost during 2D Fourier transformation. One can only conclude that there is any defect in the structure. But the main advantage of this method from numerical point of view is the fact that to obtain this type of graphs there is no need to perform time consuming transient analysis, but only frequency analysis, which significantly reduces the computation time.

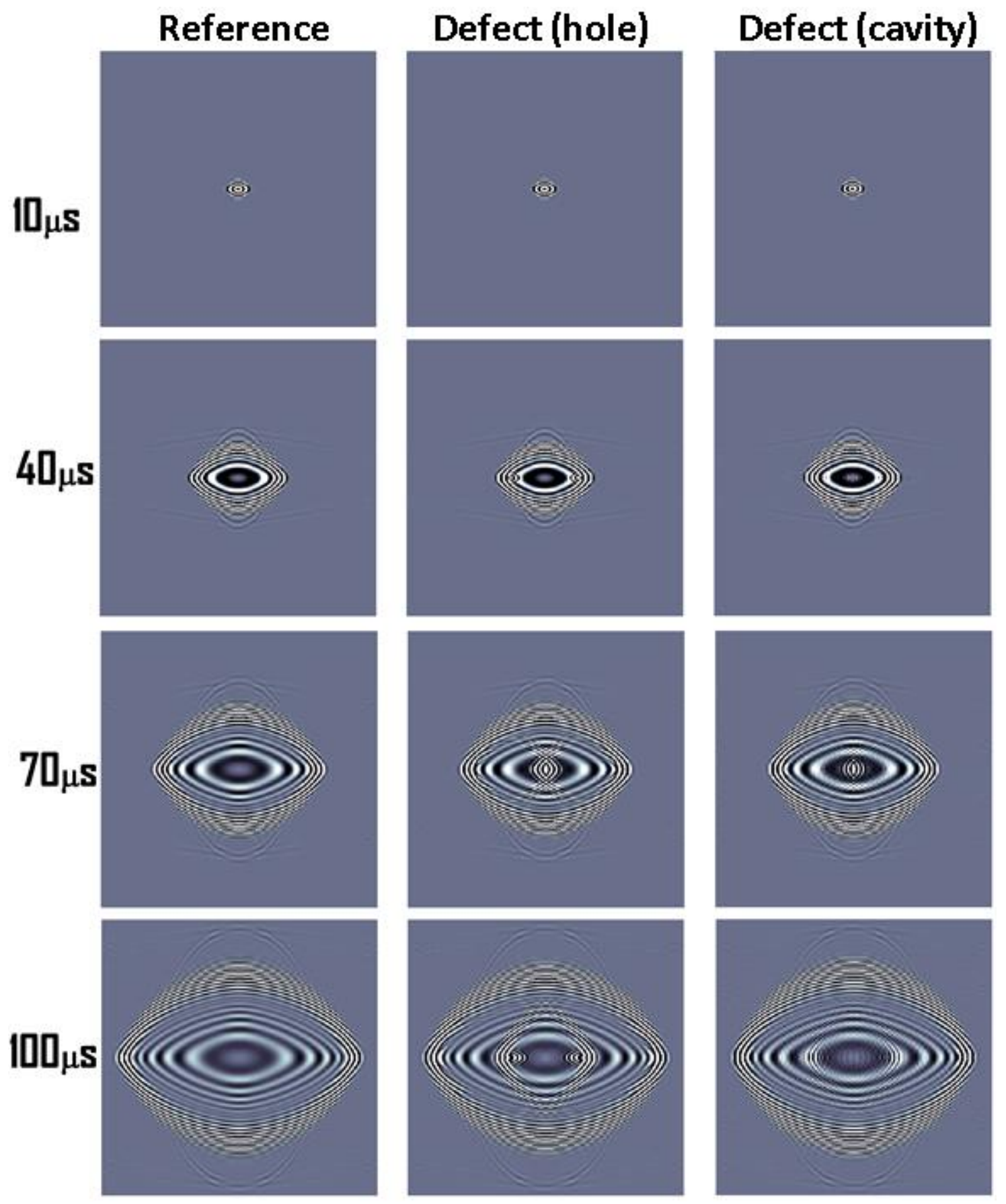

Fig - 10: Comparison of displacement field in CFRP plates with and without flaws. 

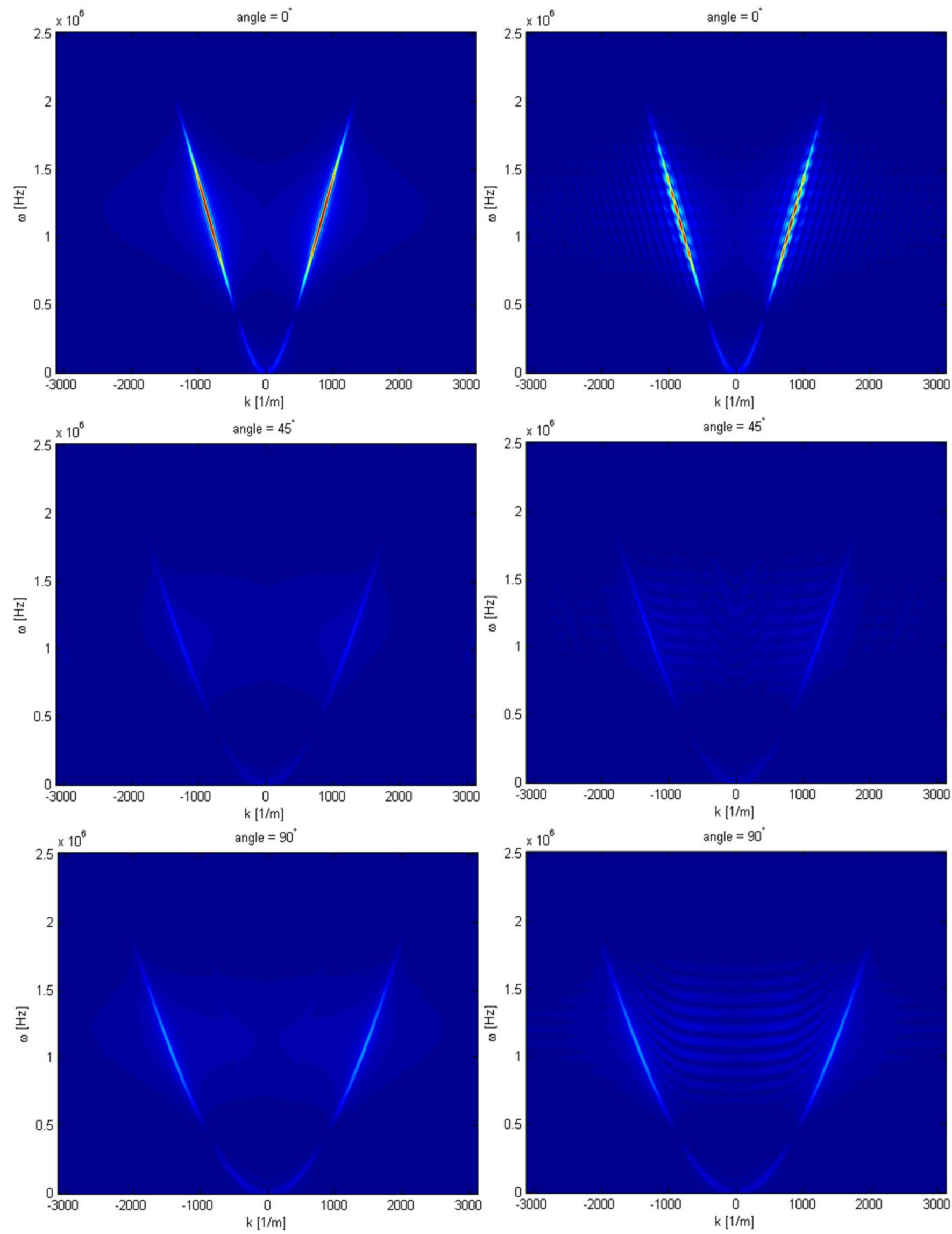

Fig - 11: Dispersion charts for undamaged and damaged CFRP plate for different angles 


\subsection{Spectrogram Method}

One further approach to detect damages in composite plates by means of ultrasonic testing is time-frequency analysis. In this paper a short-time Fourier transform and wavelet analysis will be briefly discussed. The short-time Fourier transform allows to represent recorded amplitude signal in both time and frequency domain through time windowing function. Based on obtained A-scans for different sensor locations one can calculate so called spectrogram. As in earlier presented methods the sensitivity of this technique is linked with the position of the sensor. Series of figures show selected results for undamaged and damaged (with hole) plates:

- Fig. 12 shows results for sensor position: $\mathrm{x}=0 \mathrm{~mm}$, $\mathrm{y}=0 \mathrm{~mm}, \mathrm{z}=1 \mathrm{~mm}$ (sensor is located on the top surface in the middle of the plate and it coincide with the position of excitation area),

- Fig. 13 shows results for sensor position: $x=200 \mathrm{~mm}$, $\mathrm{y}=0 \mathrm{~mm}, \mathrm{z}=1 \mathrm{~mm}$ (in this case flaw is located on the line, which connects the middle of the plate and sensor),

- Fig. 14 shows results for sensor position: $\mathrm{x}=100 \mathrm{~mm}$, $\mathrm{y}=100 \mathrm{~mm}, \mathrm{z}=1 \mathrm{~mm}$ (flaw is not situated on the line connecting transmitter and receiver),

- Fig. 15 shows results for sensor position: $x=0 \mathrm{~mm}$, $\mathrm{y}=200 \mathrm{~mm}, \mathrm{z}=1 \mathrm{~mm}$ (ditto).
Spectrograms were calculated by means of MATLAB function spectrogram with Hamming window of length 256 samples. The number of samples that each segment overlaps was set to 250 , the FFT length was set to 256 samples and the sampling frequency was $10 \mathrm{MHz}$. Again, one can see that the possibility that fault is detected depends on the location of the sensor. In first case (Fig. 12), where receiver position was the same as transmitter position, the reflection from the defect is sharp and it is very easy to distinguish between damaged and undamaged plate. For the second presented results (Fig. 13), where flaw is located on the line connecting transmitter and receiver, in case of undamaged plate first maximum of spectrogram intensity comes from symmetric Lamb mode $s_{0}$ and second comes from the antisymmetric Lamb mode $a_{0}$. In the case of damaged plate such distinction cannot be easily made. In two next cases (Figs. 14 and 15) the interpretation of the result is not so straightforward. Results depicted in Fig. 14 are obtained on the basis of signal received in point $(100 \mathrm{~mm}, 100 \mathrm{~mm}$, $1 \mathrm{~mm}$ ). The additional maximum of intensity for frequency approximately equal to $1 \mathrm{MGHz}$ for damaged plate is visible. Also distribution of spectrogram intensity is different in these two cases. Similar, non-trivial to interpret results were obtained in fourth case of sensor location (Fig. 15), which confirms that the efficiency of detection of damages depends on the relative position of the transmitter and receiver.
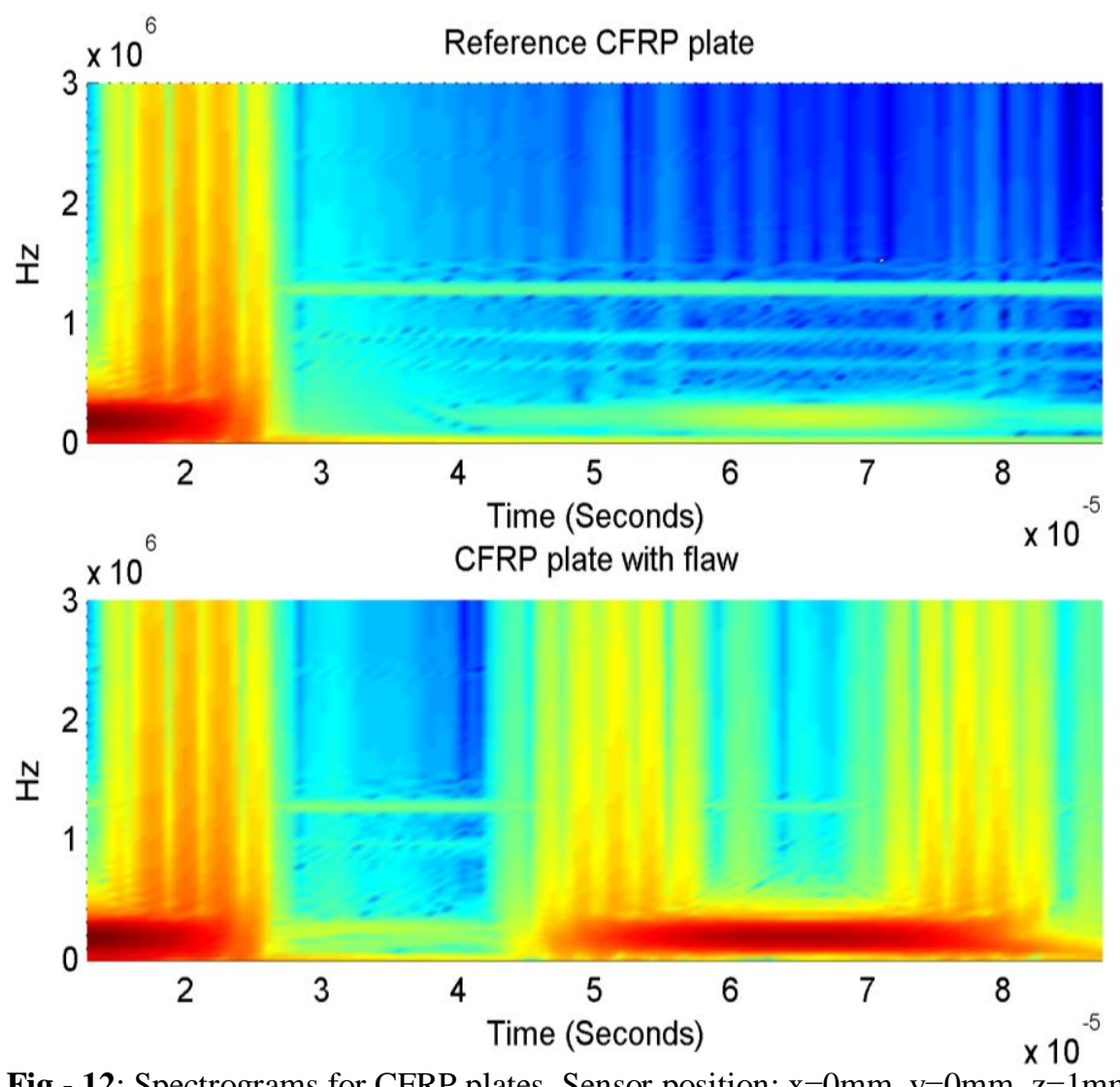

Fig - 12: Spectrograms for CFRP plates. Sensor position: $x=0 \mathrm{~mm}, y=0 \mathrm{~mm}, z=1 \mathrm{~mm}$ 


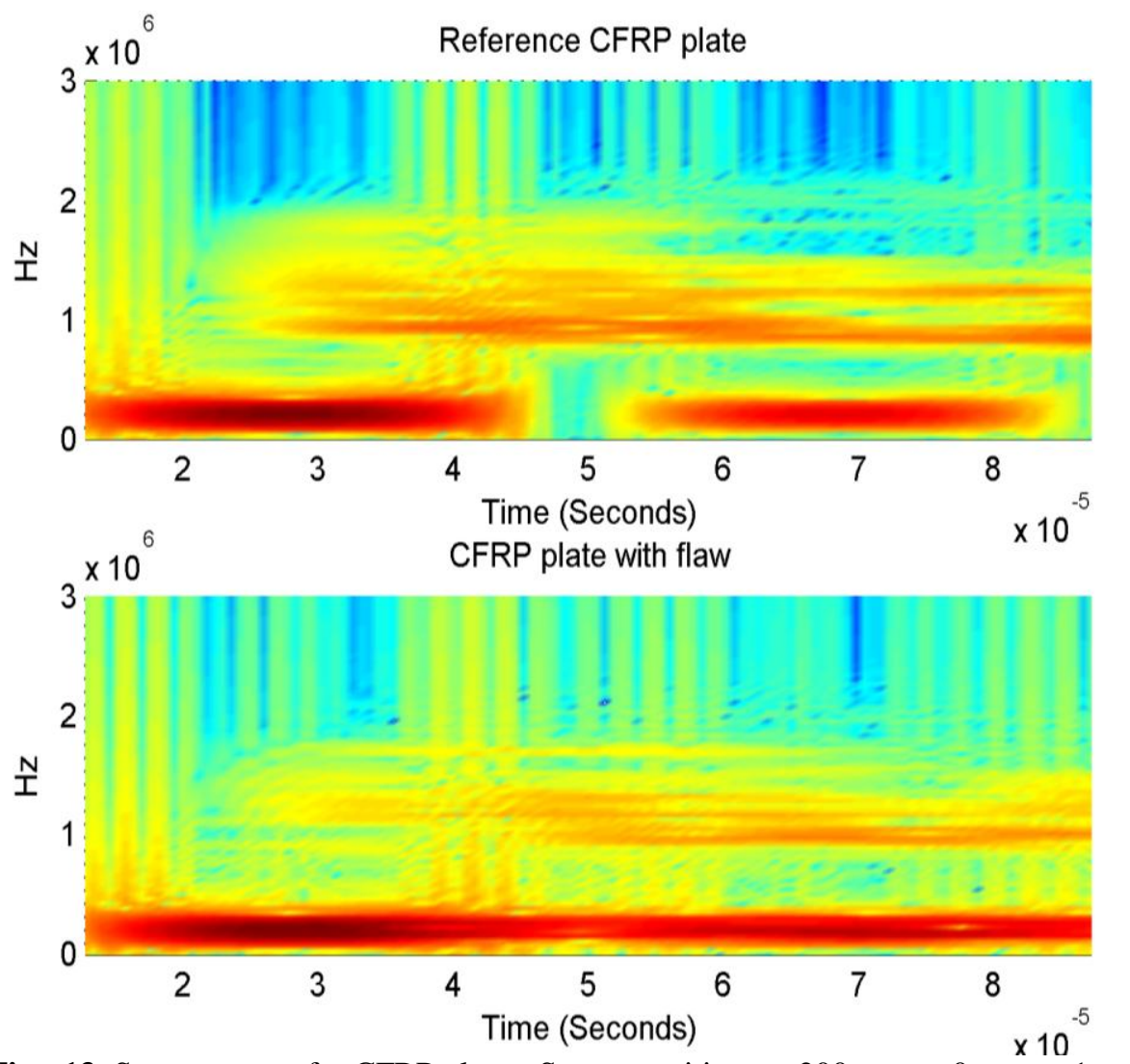

Fig - 13: Spectrograms for CFRP plates. Sensor position: $x=200 \mathrm{~mm}, y=0 \mathrm{~mm}, z=1 \mathrm{~mm}$
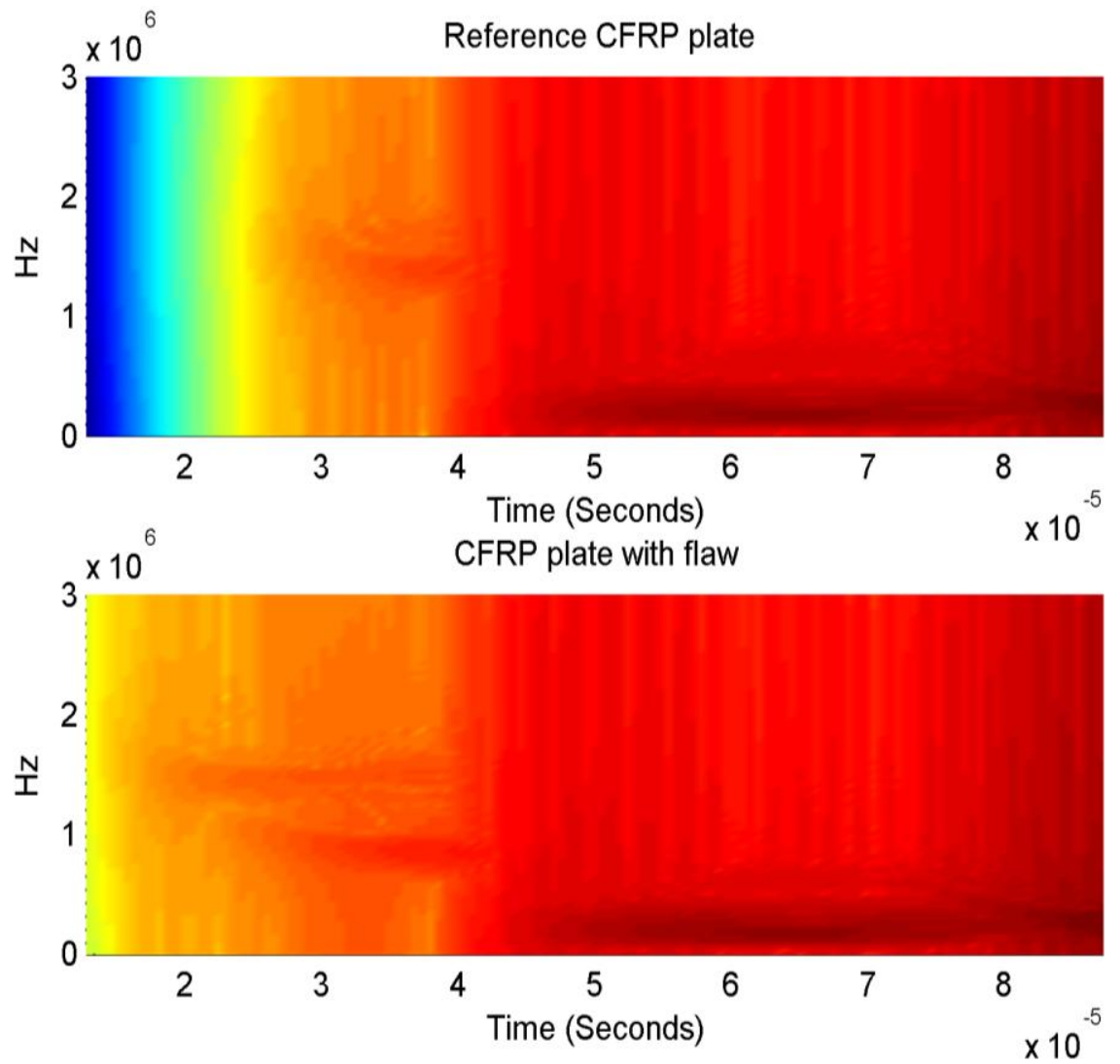

Fig - 14: Spectrograms for CFRP plates. Sensor position: $x=100 \mathrm{~mm}, y=100 \mathrm{~mm}, z=1 \mathrm{~mm}$ 

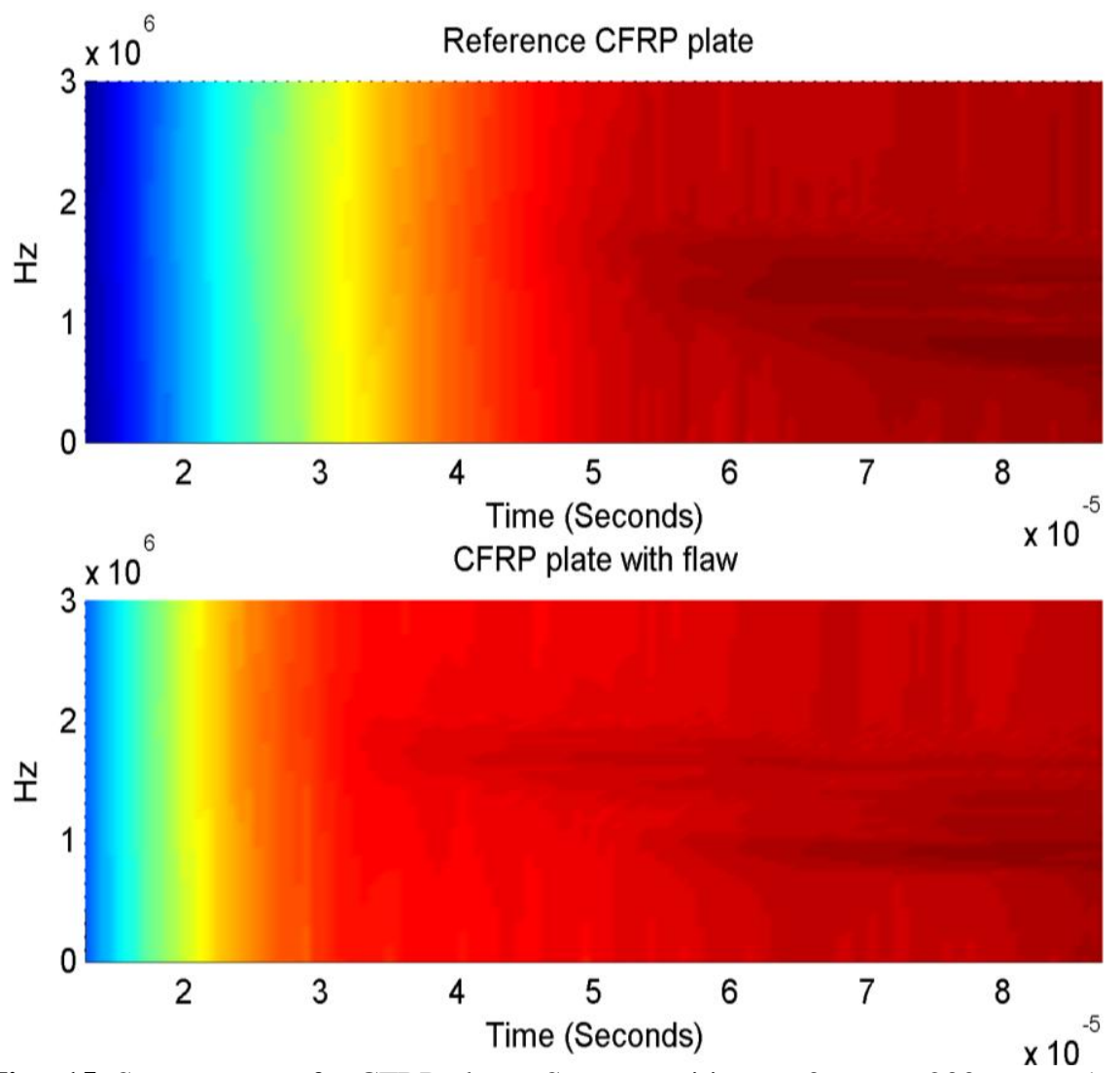

Fig - 15: Spectrograms for CFRP plates. Sensor position: $x=0 \mathrm{~mm}, y=200 \mathrm{~mm}, z=1 \mathrm{~mm}$

\subsection{Energy Plots}

Comparing the results for different receiver positions one can observe that the intensity of spectrograms, which is proportional to the energy of the signal, is different. To show this relation the normalized maximum energy of wave amplitude for different angles (angles between fibers direction and receiver position) can be plotted in polar coordinates for undamaged and damaged plates: Fig. 16 and Fig. 17, respectively. Due to the flaw the curve shown in Fig. 17 is disturbed in relation to the curve for undamaged CFRP plate. Thus, by comparing obtained results with the reference graph (Fig. 16) one can assessed whether the tested structure is damaged.

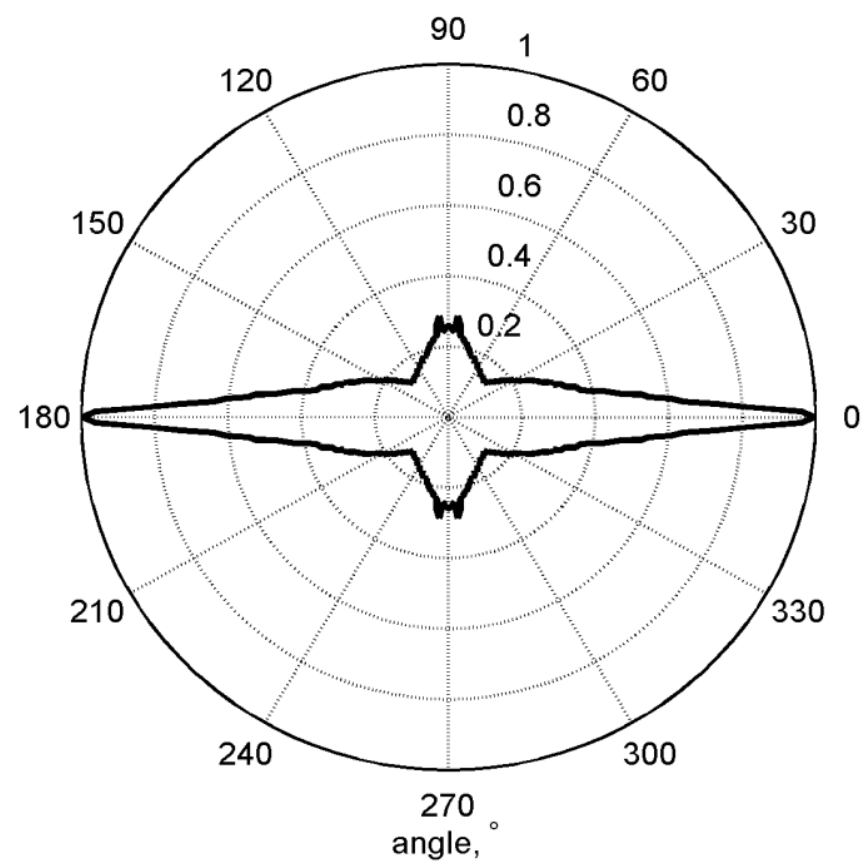

Fig - 16: Normalized maximum energy of wave or FFT amplitude for different angles - undamaged plate 


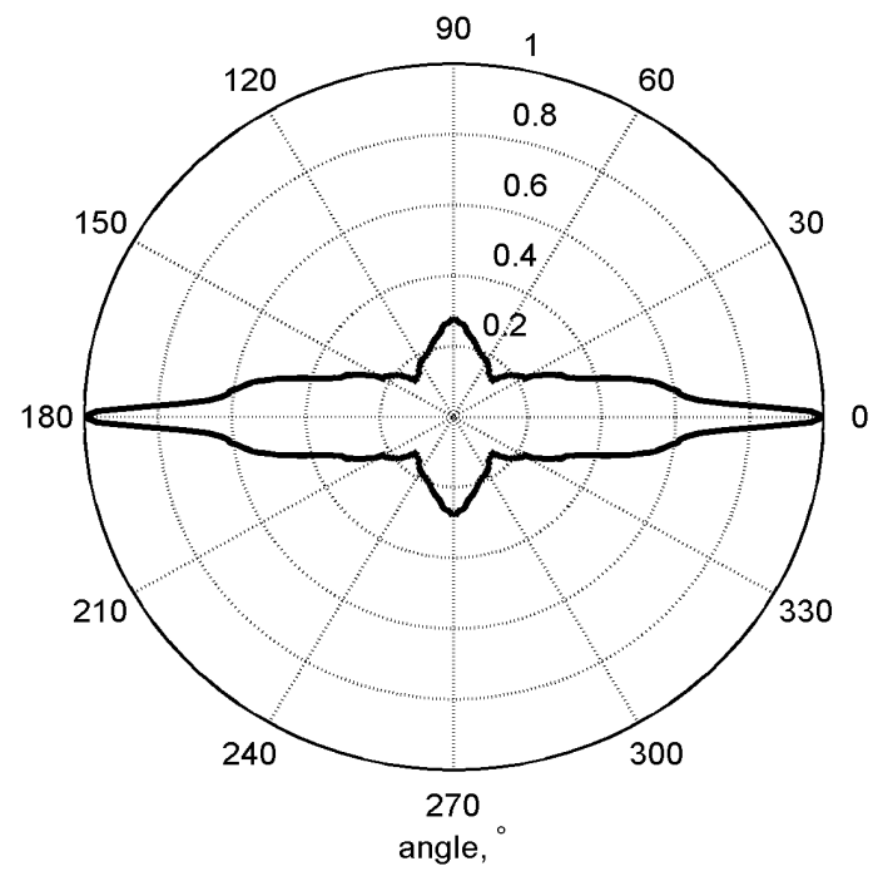

Fig - 17: Normalized maximum energy of wave or FFT amplitude for different angles - damaged plate (with hole)

\subsection{Wavelet Analysis}

Second mentioned time-frequency analysis is wavelet transform. The wavelet transform allows to represent recorded signal in both time and frequency domain. The wavelets were found to be more sensitive damage detection method than other techniques (Fourier transform, amplitude response, etc.) [20]. Many authors use Morlet and Daubechies wavelet bases for decomposition of a Lambwave response [21, 22]. In this case based on recorded A-scans one can calculate so called scalograms. In the course of numerical studies scalograms were calculated by means of MATLAB function $c w t$, which performs continuous 1-D wavelet transform. The continuous wavelet coefficients of the signal were computed at real, positive scales $1,2, \ldots, 256$, using fourth order Daubechies wavelet.
The scalograms are presented in Fig. 18 for reference CFRP plate and in Fig. 19 for damaged CFRP plate (case with the whole). Comparing obtained results with spectrograms depicted in Fig. 13 one can observe, that wavelets transform gives better localization in time than Fourier transform. In case of undamaged plate two Lamb wave modes can be easily observe. Furthermore, as in the case of spectrograms (for sensor position: $\mathrm{x}=200 \mathrm{~mm}, \mathrm{y}=0 \mathrm{~mm}, \mathrm{z}=1 \mathrm{~mm}$ ) the distinction between damaged and undamaged plate is straightforward. Also in the case of the plate with flaw reflections from the failure are clearly visible. It shows the potential of wavelet transform and possible applications in field of nondestructive testing of composite materials.

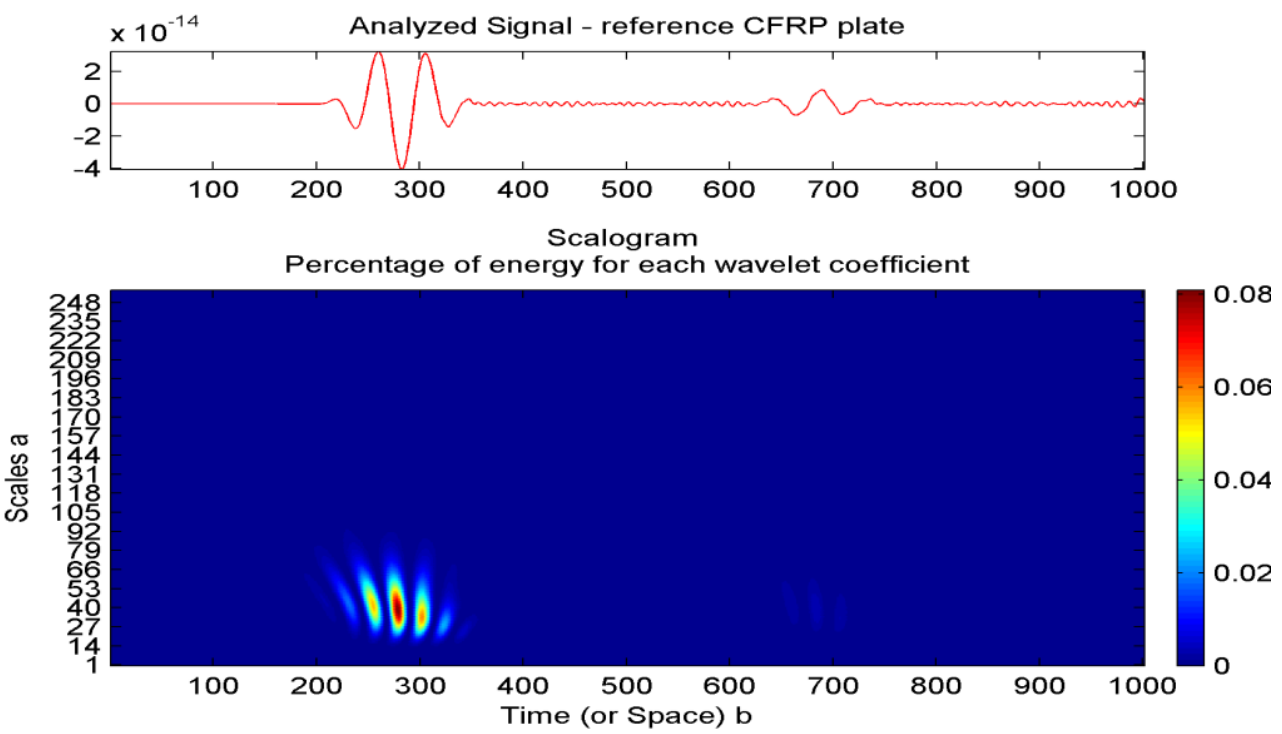

Fig - 18: Scalogram for reference CFRP plate. Sensor position: $x=200 \mathrm{~mm}, y=0 \mathrm{~mm}, z=1 \mathrm{~mm}$ 

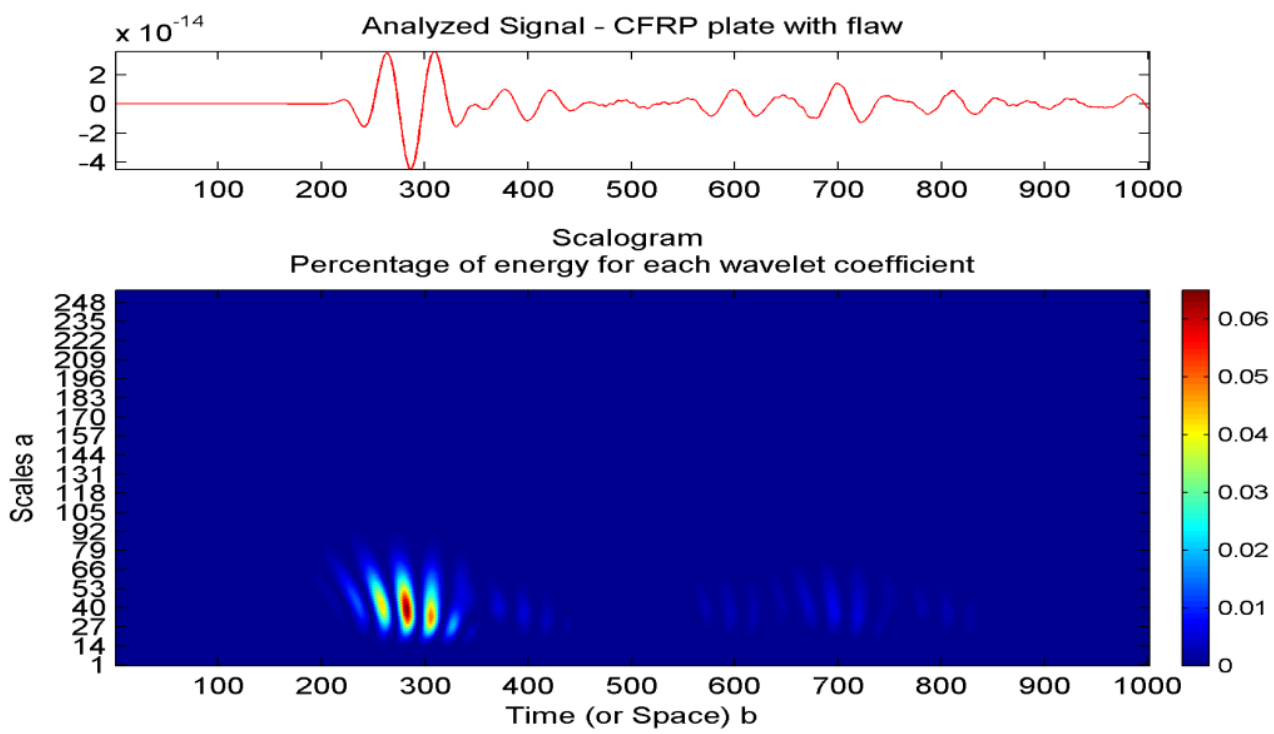

Fig - 19: Scalogram for CFRP plate with flaw (hole). Sensor position: $x=200 \mathrm{~mm}, y=0 \mathrm{~mm}, z=1 \mathrm{~mm}$

\section{CONCLUSION}

The paper has presented a numerical aspects of selected issues associated with Lamb waves propagation in CFRP plates for damage detection. Firstly it was shown that implemented finite element model gives an accurate results for isotropic material. Theoretical and numerical results agree well. It can therefore be argued that the model gives accurate results also in the case of plates made of CFRP, especially that transition between isotropic and anisotropic case is straightforward from software point of view. First obtained result for CFRP plates with simple geometry show the potential of proposed method in damage identification. Postprocessing of model's results by means of short-time Fourier transform and wavelet transform facilitates detection of flaws. It should, however, be admitted that the finite element method is not the most efficient method in case of ultrasonic wave propagation in solid materials. To achieve high accuracy of numerical results for high excitation frequency the time step and spatial resolution of mesh should be extremely fine from point of view of FEM. This leads to a huge load of computer workstations and sometimes results in unacceptable computation time. However, the development of computers and increasing the efficiency of numerical algorithms allows believing that in the near future, these problems will be overcome. One of the promising method is a hybrid spectral-element/finiteelement time-domain method for multiscale simulations.

\section{ACKNOWLEDGEMENTS}

Financial support of Structural Funds in the Operational Programme - Innovative Economy (IE OP) financed from the European Regional Development Fund - Project "Modern material technologies in aerospace industry", No. POIG.01.01.02-00-015/08-00 is gratefully acknowledged.

\section{REFERENCES}

[1]. Thori P., Sharma P., Bhargava M., "An Approach of Composite Materials in Industrial Machinery: Advantages, Disadvantages and Applications", IJRET, 2(12), 2013, pp. 350-355

[2]. Knor G., Holnicki-Szulc J., "Modelling Propagation of Lamb Waves in CFRP Plates for Damage Detection", 39th Solid Mechanics Conference SolMech, 2014, pp. 229-230

[3]. Lamb H., "On Waves in an Elastic Plate", Proc. Roy. Soc. London, Ser. A 93, 1917, pp. 114-128

[4]. Lu Y., Ye L., Wang D., Zhong Y. and Herszberg I., "Damage detection in a large composite panel of five stiffeners using lamb wave signals", Materials Forum, 33, 2009 , pp. 136-142

[5]. Balasubramanyam R., Quinney D., Challis R.E., Todd C.P.D., "A finite-difference simulation of ultrasonic Lamb waves in metal sheets with experimental verification", Journal of Physics D: Applied Physics 29(1), 1996, pp. 147-155

[6]. Ng C.T., Veidt M., Rose L.R.F., Wang C.H., "Analytical and finite element prediction of Lamb wave scattering at delaminations in quasi-isotropic composite laminates", Journal of Sound and Vibration, 331(22), 2012, pp. 4870-4883

[7]. Liu G.R., Achenbach J. D., "Strip Element Method to Analyze Wave Scattering by Cracks in Anisotropic Laminated Plates", J. Appl. Mech. 62(3), 1995, pp.607-613 [8]. Wang S., Huang S., Zhao W., "Simulation of Lamb wave's interactions with transverse internal defects in an elastic plate", Ultrasonics, 51(4), 2011, 432-440

[9]. Nayfeh, A.H., "Wave Propagation in Layered Anisotropic Media with Applications to Composites", Elsevier, 1995

[10]. Ostachowicz W., Kudela P., Krawczuk M., Zak A., "Guided Waves in Structures for SHM: The Time - domain Spectral Element Method", Wiley, 2012

[11]. Yim H., Sohn Y., "Numerical simulation and visualization of elastic waves using mass-spring lattice 
model", IEEE Transactions on Ultrasonics, Ferroelectrics and Frequency Control, 47(3), 2000, pp. 549-558

[12]. Wilcox P.D., Velichko A., Drinkwater B.W., Croxford A.J., "Scattering of plane guided waves obliquely incident on a straight feature with uniform cross-section", J. Acoust. Soc. Am., 128(5), 2010, pp. 2715-2725

[13]. Raišutis R., Kažys R., Žukauskas E., Mažeika L., Jankauskas A., Burnham K., "Application of ultrasonic guided waves for investigation of structural components of tidal power plants", CompNDT 2011, Ultrasonic Testing of Composite Material by Guided Waves, 2011

[14]. Shannon C.E., "Communication in the presence of noise", Proc. Institute of Radio Engineers, vol. 37, no. 1, 1949, pp. 10-21

[15]. Rose J. L., "Ultrasonic Waves in Solid Media", Cambridge University Press, 1999

[16]. COMSOL Inc., "Resolving time-dependent waves", COMSOL knowledgebase, 2014

[17]. Courant R., Friedrichs K., Lewy H., "On the partial difference equations of mathematical physics", IBM Journal, pp. 215-234, 1967

[18]. Horaa P., Cervena O., "Determination of Lamb wave dispersion curves by means of Fourier transform", Applied and Computational Mechanics 6, 2012, pp. 5-16

[19]. Sause M.G.R., Horn S., "Influence of specimen geometry on acoustic emission signals in fiber reinforced composites: FEM-simulations and experiments", Conf. Proc. 29th European Conf. on Acoustic Emission Testing, Vienna, Austria, 2010

[20]. Paget, C.A., "Contribution to active health monitoring of aerospace structures by embedded piezoceramic transducers", PhD Report, Valenciennes University, 2001

[21]. Paget, C.A., Grondel S., Levin K., Delebarre C., "Damage assessment in composites by Lamb waves and wavelet coefficients", Smart Materials and Structures 12(3), 2003, pp. 393-402

[22]. Staszewski W.J., Pierce S.G., Worden K., Philp W.R., Tomlinson G.R., Culshaw B., "Wavelet signal processing for enhanced Lamb-wave defect detection in composite plates using optical fiber detection”, Opt. Eng. 36, 1997, pp. $1877-88$

\section{BIOGRAPHY}

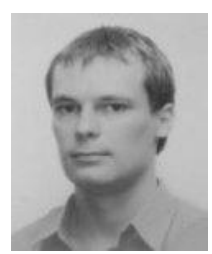

Grzegorz Knor, has received his masters from the Faculty of Physics at the University of Warsaw. Currently working as assistant at Institute of Fundamental Technological Research Polish Academy of Sciences 